\title{
Severidad y Duración del Estrés Calórico en Terneras y Vaquillas de las Principales Localidades de Lechería Intensiva del Departamento de Lima, Perú
}

\author{
Severity and Duration of Heat Stress in Calves and Heifers in the Main Intensive \\ Dairy Localities of the Department of Lima, Peru
}

Luis Felipe Ruiz G. ${ }^{1}$, Rocío Sandoval M. ${ }^{1,3}$, Joe Pizarro P. ${ }^{1}$, Fernando Carcelén C. ${ }^{2}$

\section{Resumen}

\begin{abstract}
El objetivo del estudio fue determinar la severidad y duración del estrés calórico en terneras y vaquillas lecheras de las cinco principales localidades de lechería intensiva del departamento de Lima, Perú (Végueta, Huacho, Huaral, Lima y Cañete) durante el periodo 2010-2013. Se determinó el índice temperatura-humedad sobrecarga (ITH sobrecarga) y la duración del estrés calórico por mes y localidad, y el porcentaje de días del año donde el ITH sobrecarga fue superior a 0 . Los resultados demostraron que el ITH sobrecarga mensual fue mayor que cero en todos los meses del año para las vaquillas. Asimismo, febrero fue el mes donde el estrés calórico se presentó con mayor severidad y duración, tanto en vaquillas como en terneras, y Cañete fue la localidad con el mayor ITH sobrecarga para terneras y vaquillas. Con respecto a la duración del estrés calórico, las vaquillas y las terneras pasaron cerca de 10 y 5 horas diarias, respectivamente, expuestas a estrés calórico en los meses de verano. Las vaquillas de Végueta y Cañete pasaron el 86 y $83 \%$ de días del año con estrés calórico, respectivamente, mientras que las terneras de Cañete pasaron el $47 \%$ de los días con estrés calórico. Se concluye que en las localidades de Cañete y Végueta, a diferencia de las otras localidades, se presenta con mayor severidad y duración el estrés calórico en las vaquillas, en tanto que solo ocurre en Cañete para el caso de las terneras.
\end{abstract}

Palabras clave: estrés calórico; terneras; vaquillas; Lima; severidad; duración

\footnotetext{
${ }^{1}$ Clínica de Animales Mayores, ${ }^{2}$ Laboratorio de Bioquímica, Nutrición y Alimentación Animal, Facultad de Medicina Veterinaria, Universidad Nacional Mayor de San Marcos, Lima, Perú

${ }^{3}$ E-mail: rocio.sandoval@unmsm.edu.pe
}

Recibido: 8 de diciembre de 2016

Aceptado para publicación: 20 de abril de 2017 
The aim of this study was to determine the severity and duration of heat stress in dairy calves and heifers of the five main dairy localities in Lima, Peru (Végueta, Huacho, Huaral, Lurin, Cañete) during the period 2010-2013. The temperature-humidity index load (ITH load) and the duration of the heat stress per month and location were evaluated. In addition, the percentage of days in the year in which ITH load was greater than 0 . The results showed that the ITH load was greater than zero in all months of the year for heifers. Also, February was the month where heat stress occurred with greater severity and duration, both in heifers and in calves and Cañete was the locality with the highest ITH overload for calves and heifers. Regarding the duration of heat stress, heifers and calves spent about 10 and 5 hours a day, respectively, exposed to caloric stress during the summer months. Heifers in Végueta and Cañete spent 86 and $83 \%$ of days of the year with heat stress, respectively, while calves in Cañete spent $47 \%$ of the days in heat stress. It is concluded that heat stress in heifers in the localities of Cañete and Végueta, as compared with the other localities, occurred with greater severity and duration, whereas it only occurs in Cañete for calves.

Key words: heat stress; calves; heifers; Lima; severity; duration

\section{INTRODUCCIÓN}

El mantenimiento de la temperatura corporal en un individuo se conoce como homeotermia y esta función es decisiva para mantener las funciones corporales normales sin alterar otras funciones fisiológicas (Jiménez, 2005). La homeotermia es el resultado de equilibrar el calor producido por el organismo con la pérdida o ganancia de calor desde el ambiente que lo rodea (Mader et al., 2006; Martínez, 2006; Ronald et al., 2016). El calor transmitido desde el ambiente es básicamente el resultado de la temperatura ambiental y la humedad relativa (St-Pierre et al., 2003; West, 2003; Martínez, 2006). El índice temperatura-humedad (ITH) representa el efecto combinado de la temperatura ambiental y humedad relativa y se ha utilizado como indicador de estrés calórico en el hombre y los animales (St-Pierre et al., 2003; West, 2003).

El ITH óptimo es aquel que exige el mínimo consumo de alimento para mantener la temperatura corporal dentro de los límites normales y maximizar el crecimiento y la pro- ducción. El rango de ITH óptimo se conoce como zona termoneutral, el cual varía de acuerdo a la especie, raza y estado productivo. Cuando los animales están por debajo o por encima de su zona termoneutral, su ritmo metabólico aumenta o disminuye, respectivamente (Jiménez, 2005; Mujibi et al., 2010).

El ITH óptimo tienen un estrecho rango en especies homeotérmicas (Katschinski, 2004). El estrés calórico se inicia cuando el ITH del ambiente supera el límite mayor del ITH óptimo, punto que se le conoce como el ITH umbral. Este punto en las terneras y vaquillas lecheras es de 72 y 77 unidades respectivamente (St-Pierre et al., 2003). Se produce estrés calórico en un animal, cuando no se puede disipar el excedente de la suma del calor producido por su metabolismo y del calor del medio ambiente, generando hipertermia (Aréchiga-Flores y Hansen, 2003; West, 2003; Ronald et al., 2016).

Los efectos ambientales han sido ampliamente reconocidos como factores que afectan la productividad y la eficiencia reproductiva de los animales de producción a nivel mundial (St-Pierre et al., 2003, 
Amundson et al., 2006; Bolocan, 2009). Cuando el periodo de estrés es corto se observa una leve disminución en la producción de leche en el ganado bovino, pero si el periodo de estrés es prolongado la producción y reproducción de los animales puede sufrir serias consecuencias (St-Pierrre et al., 2003; Arias y Mader, 2010). El ganado vacuno responde al estrés por calor reduciendo el consumo de alimento e incrementando el consumo de agua; asimismo, ocurre pérdida de agua por evaporación, y aumento de la frecuencia respiratoria y la temperatura corporal (Thatcher, 1974; Wise et al., 1988; West et al., 2003; Wheelock et al., 2010: BrownBrandl et al., 2016).

E1 ITH presenta una función senoidal en el transcurso del día. Con esta base, StPierre et al. (2003) propusieron nuevos índices para estimar el estrés calórico; entre ellos, la duración del estrés calórico y la severidad del estrés calorico que puede medirse con el ITH sobrecarga. El primero es el tiempo que el ITH excede el ITH umbral, y el segundo es el integral de la curva senoidal de ITH sobre el ITH umbral, el cual representa el calor acumulado por encima del umbral durante las 24 horas del día (St-Pierre et al., 2003). Estos indicadores permiten predecir los efectos adversos del estrés calórico sobre la producción y reproducción del ganado (St-Pierre et al., 2003).

Diversos estudios evidencian que las cuencas lecheras del norte y centro del Perú presentan condiciones climatológicas propicias para ocasionar estrés calórico en el ganado durante los meses de verano (Rodríguez et al., 2005; Domínguez, 2008; Contreras, 2009; Sandoval et al., 2017); sin embargo, la severidad y la duración del estrés calórico en las terneras y las vaquillas lecheras no han sido medidas. El departamento de Lima posee importantes localidades de producción lechera (INEI, 2012) y sus características climatológicas de Lima hacen posible la hipótesis de que el estrés calórico no solo afecte a los animales en producción, sino que también esté presente en las terneras y las vaquillas. Por lo tanto, el objetivo de este trabajo fue determinar la severidad y duración del estrés calórico en las terneras y vaquillas lecheras de las cinco principales localidades de lechería intensiva del departamento de Lima, mediante los indicadores del estrés calórico.

\section{Materiales y Métodos}

El estudio se realizó en cinco localidades de importancia para la ganadería lechera intensiva del departamento de Lima, Perú (INEI, 2012), entre enero de 2010 y agosto de 2013. Las localidades fueron Végueta, Huacho, Huaral, Lima y Cañete.

Se recolectaron datos de los registros meteorológicos diarios registrados por el Servicio Nacional de Meteorología e Hidrografía (SENAMHI) durante el periodo del estudio de las estaciones meteorológicas cercanas (zona Végueta: Estación Camay 000532; zona Huacho: Estación Alcantarilla - 000501; zona Huaral: Estación Donoso 000546; zona Lurín: Estación Villa María del Triunfo - 472A0766; zona Cañete: Estación Pacarán - 000638).

Se recolectaron los registros meteorológicos diarios $(\mathrm{n}=6512)$ y se utilizaron para determinar el tiempo que las terneras y las vaquillas estuvieron sometidas a estrés calórico, así como para estimar el ITH sobrecarga para las terneras y las vaquillas por mes y por localidad. Además, se calculó el porcentaje de días en los cuales los valores de ITH sobrecarga fueron mayores a cero por localidad.

Se asumió que la temperatura y la humedad son variables anticíclicas durante el transcurso del día (Ravagnolo et al., 2000; St-Pierre et al., 2003) y que el ITH sigue una función senoidal perfecta (St-Pierre et al., 2003). El ITH sobrecarga y el estrés calórico fueron calculados según las ecuaciones descritas por St-Pierre et al. (2003), consi- 
Cuadro 1. Valores promedios y límites de confianza al 95\% $(\mathrm{x} \pm \mathrm{LC})$ de la sobrecarga de calor (ITH sobrecarga) y la duración (D) del estrés calórico anual en las terneras (0-1 año) y vaquillas (1-2 años) lecheras de cinco localidades del departamento de Lima (2010-2013)

\begin{tabular}{llccccccc}
\hline & \multicolumn{4}{c}{ Terneras } & \multicolumn{5}{c}{ Vaquillas } \\
\cline { 2 - 9 } & \multicolumn{1}{c}{ ITH sobrecarga } & \multicolumn{2}{c}{$\mathrm{D}$} & \multicolumn{1}{c}{ ITH sobrecarga } & $\mathrm{D}$ \\
\cline { 2 - 9 } & $\mathrm{x}$ & $\pm \mathrm{LC}$ & $\mathrm{x}$ & $\pm \mathrm{LC}$ & $\mathrm{x}$ & $\pm \mathrm{LC}$ & $\mathrm{x}$ & $\pm \mathrm{LC}$ \\
\hline Cañete & $6.68^{\mathrm{a}}$ & 0.57 & $3.04^{\mathrm{a}}$ & 0.20 & $33.46^{\mathrm{a}}$ & 1,71 & $7.92^{\mathrm{a}}$ & 0.27 \\
Huacho & $1.19^{\mathrm{b}}$ & 0.20 & $0.94^{\mathrm{b}}$ & 0.12 & $14.59^{\mathrm{b}}$ & 1,18 & $4.72^{\mathrm{b}}$ & 0.29 \\
Huaral & $0.98^{\mathrm{b}}$ & 0.18 & $0.79^{\mathrm{b}}$ & 0.12 & $11.92^{\mathrm{c}}$ & 1,08 & $3.86^{\mathrm{c}}$ & 0.27 \\
Lurín & $0.21^{\mathrm{c}}$ & 0.06 & $0.28^{\mathrm{c}}$ & 0.06 & $9.26^{\mathrm{d}}$ & 0.90 & $3.79^{\mathrm{c}}$ & 0.29 \\
Végueta & $0.00^{\mathrm{c}}$ & 0.00 & $0.01^{\mathrm{c}}$ & 0.00 & $7.78^{\mathrm{b}}$ & 0.37 & $5.16^{\mathrm{b}}$ & 0.14 \\
\hline a,b,c Superíndices diferentes dentro de columnas &
\end{tabular}

$a, b, c$ Superíndices diferentes dentro de columnas indican diferencia significativa $(p<0.05)$

Cuadro 2. Número de días $(\% \pm \mathrm{LC} 95 \%)^{1}$ con estrés calórico en terneras (0-1 año) y vaquillas (1-2 años) lecheras de cinco localidades del departamento de Lima (2010-2013)

\begin{tabular}{lcc}
\hline & Vaquillas & Terneras \\
\cline { 2 - 3 } & \pm LC & $\% \pm$ LC \\
\hline Cañete & $83.2 \pm 2.0^{\mathrm{b}}$ & $47.1 \pm 2.7^{\mathrm{a}}$ \\
Huacho & $49.4 \pm 2.7^{\mathrm{c}}$ & $17.6 \pm 2.1^{\mathrm{b}}$ \\
Huaral & $41.3 \pm 2.6^{\mathrm{c}}$ & $15.7 \pm 2.0^{\mathrm{b}}$ \\
Lurín & $38.6 \pm 2.7^{\mathrm{c}}$ & $6.6 \pm 1.4^{\mathrm{c}}$ \\
Végueta & $86.0 \pm 1.9^{\mathrm{a}}$ & $0.3 \pm 0.3^{\mathrm{d}}$ \\
\hline
\end{tabular}

a,b,c Superíndices diferentes dentro de columnas indican diferencia significativa $(p<0.05)$

${ }^{1}$ Límite de confianza al 95\%

derando un ITH umbral de 72 para vaquillas y 77 para terneras.

Los valores de ITH sobrecarga y la duración del estrés calórico se evaluaron mediante la prueba de Friedman, tanto para cada localidad como para los meses del año.
Para determinar diferencias entre promedios se utilizó la prueba de comparación por pares. Asimismo, el porcentaje de días con valores de ITH sobrecarga mayor que cero en los dos grupos de animales fue analizado mediante un modelo de regresión logística binaria, donde la variable independiente fue la localidad, estimando los coeficientes de regresión para cada combinación para determinar diferencias estadísticas entre grupos. Los datos fueron analizados mediante el paquete estadístico SPSS v. 22.0 para Windows. En todos los análisis se empleó un nivel de significancia de $5 \%$.

\section{Resultados}

El ITH sobrecarga promedio mensual fue mayor que cero para las vaquillas en todos los meses del año (Figura 1), siendo mayor en los meses de verano en comparación con los meses de invierno $(\mathrm{p}<0.05)$. Así también, se encontró que el mes con mayor ITH sobrecarga fue febrero $(\mathrm{p}<0.05)$. Por otro lado, el ITH sobrecarga en las terneras fue mayor a cero desde diciembre hasta abril, pero menor que en vaquillas $(p<0.05)$. 


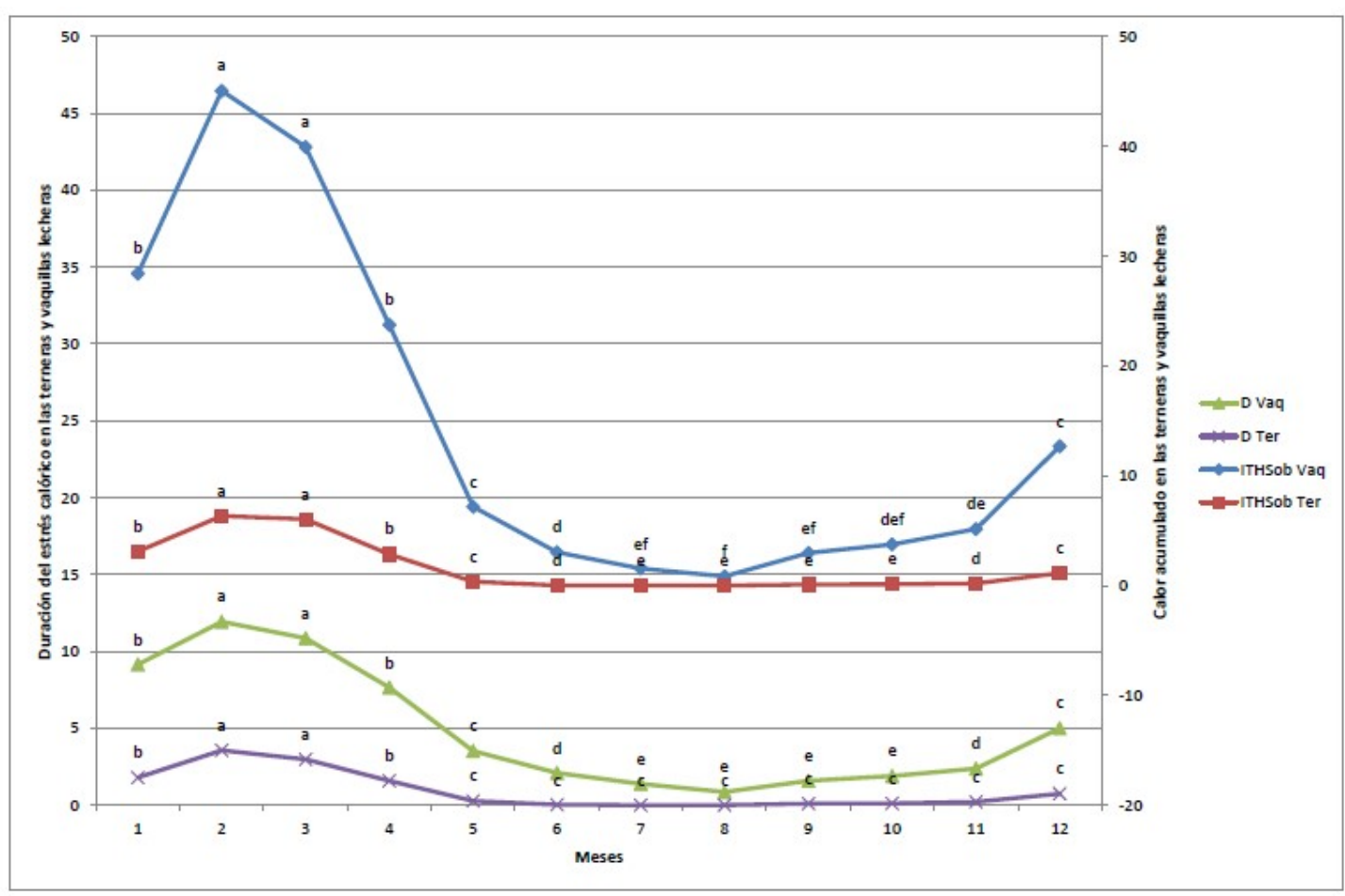

Figura 1. Valores promedios de la sobrecarga de calor (Índice Temperatura-Humedad - ITH sob) y la duración (D) del estrés calórico en terneras (Ter) y vaquillas (Vaq) lecheras por mes en el departamento de Lima durante el periodo 2010-2013. Letras diferentes indican diferencia significativa $(\mathrm{p}<0.05)$ dentro de cada variable

E1 ITH sobrecarga en las vaquillas fue significativamente superior en la localidad de Cañete (ITH sobrecarga: 33.46) en comparación con las demás localidades $(\mathrm{p}<0.05)$, llegando a ser 2 a 3 veces mayor (Cuadro 1). Así mismo, el ITH sobrecarga en las terneras de la localidad de Cañete (6.68) fue mayor que en las otras localidades, donde prácticamente estuvo ausente en las localidades de Lurín y Végueta (Cuadro 1).

Las vaquillas, en los meses de verano, pasan cerca de 10 horas diarias expuestas a estrés calórico (ITH sobrecarga mayor que cero) en los meses de verano (febrero y marzo) $(p<0.05$, Figura 1). Las terneras, por otro lado, no pasan más de cinco horas expuestas al estrés calórico en esos meses (Figura 1). Considerando las localidades, las vaquillas de Cañete pasaron cerca de ocho horas diarias bajo estrés calórico, seguido de las localida- des de Végueta y Huacho donde pasaron cinco horas expuestas al estrés calórico. En el caso de las terneras, la duración del estrés calórico en la localidad de Cañete fue de tres horas, siendo una hora menos en las otras localidades.

En el Cuadro 2 se presenta el porcentaje de días del año, según localidad, que las terneras y vaquillas están expuestas a estrés calórico. Las vaquillas de Cañete y Végueta están sometidas a este estrés el 83.2 y $86.0 \%$ de los días del año, respectivamente, a diferencia de otras localidades en las que menos del $50 \%$ de los días del año están sometidas a algún grado de estrés calórico. En el caso de las terneras, las que se encuentran en Cañete se encuentran sometidas el $47 \%$ de los días del año al estrés calórico, mientras que en Végueta no presentaron estrés calórico. 


\section{Discusión}

Los resultados del estudio demuestran que las vaquillas y terneras de varias zonas de lechería intensiva del departamento de Lima se encuentran potencialmente sometidas a estrés calórico. Asimismo, se observa que el estrés calórico no solo ocurre en los meses de verano, como es considerado por la mayoría de ganaderos y profesionales dedicados a la ganadería lechera, sino que es un problema que está presente en la mayoría de los meses del año, solo que se presenta con mayor severidad durante el verano. Esto se debe principalmente a que la temperatura máxima promedio del día se encuentra entre 20 y $29^{\circ} \mathrm{C}$, lo que junto a una alta humedad mínima promedio de $69-80 \%$, ocasionan un ITH máximo de 67 a 78. Como se puede observar, el ITH máximo se encuentra muy cerca del ITH umbral para vaquillas que es de 72 (St-Pierre et al., 2003).

En el presente trabajo se encontró que las localidades con mayor estrés calórico en términos de duración y severidad fueron Végueta y Cañete, respectivamente. En Végueta, las vaquillas lecheras pasan un $86 \%$ de los días del año con algún grado de estrés calórico, en tanto que en Cañete se observa mayor severidad de estrés calórico (ITH sobrecarga de 33.46). Esto indica que los ganaderos de estas localidades deben realizar mayores inversiones para contrarrestar los efectos adversos del estrés calórico. Estas localidades, como es de esperarse, presentan mayor riesgo de presentación de estrés calórico que las regiones sureñas de Chillán, Curicó, Osorno y Temuco en Chile (Arias y Mader, 2010).

En la localidad de Cañete, las ocho horas diarias (2980 h/año) de estrés calórico que sufren las vaquillas, equivalen a una sobrecarga de calor de 33 unidades/día, en tanto que las tres horas diarias ( $1095 \mathrm{~h} / \mathrm{anno}$ ) de estrés calórico de las terneras equivalen a una sobrecarga de calor de 7 unidades/día. Esto es comparable con lo reportado por St-
Pierre et al. (2003) en Texas, EEUU, donde las vaquillas lecheras pasan $2652 \mathrm{~h} /$ año en estrés calórico, aunque con una sobrecarga de calor mucho mayor (54 unidades/día), en tanto que en Arizona, EEUU, las terneras pasan 734 horas al año en estrés calórico, con una sobrecarga de calor de 9 unidades/día. En la localidad de Végueta, las vaquillas pasan cinco horas diarias (1825 horas/año) en estrés calórico y tuvieron una sobrecarga promedio de 8 unidades/día (2920 unidades/año). Sin embargo, a diferencia de dichos estados en EEUU, los establos lecheros de las localidades evaluadas en Lima no cuentan con sistemas adecuados para el control de los efectos del estrés calórico.

El estrés calórico tiene un impacto significativo en todas las especies de ganado y aves de corral causando pérdidas económicas y mucha preocupación en lo concerniente al bienestar animal (Brown-Brandl et al., 2016). Un estudio realizado por Franco et al. (2014) demostró que diversas razas de ganado vacuno desencadenaron respuestas termorreguladoras, pero la raza Holstein presentó respuestas termorreguladoras más intensas. Es así que considerando que la Holstein es la raza más frecuente en los establos lecheros de crianza intensiva de Lima (INEI, 2012), es preocupante la duración y severidad del estrés calórico encontrada durante los meses del verano en las localidades de Lima, siendo más alarmante aun si se suma a ello que la gran mayoría de corrales de vaquillas no cuentan con mecanismos de ventilación y baños por aspersión (Muroya, 2006).

Existen pocos estudios que han podido cuantificar el efecto negativo del estrés calórico en vaquillas criadas en la cuenca lechera de Lima (Domínguez, 2008). El incremento de ITH conlleva a un aumento de la temperatura uterina que, a su vez, incrementa la mortalidad embrionaria. Esto ha sido demostrado en vaquillas Holstein superovuladas y expuestas a estrés calórico, donde solo el $20.7 \%$ de los embriones recuperados fueron normales (Putney et al., 1988). Asimismo, se ha encontrado en vaquillas Holstein afecta- 
das por el estrés calórico entre los días 11 y 21 del ciclo estral la ocurrencia de un menor tamaño folicular, menores concentraciones de estradiol, retardo en la luteólisis y mayor frecuencia de ciclos estruales con tres ondas foliculares, aunque los niveles de progesterona fueron normales (Wilson et al., 1998). Del mismo modo, en terneras de 2 a 10 días lecheras se halló una menor concentración sérica de inmunoglobulinas (Igs) (Mohammed et al., 1991), debido a la mayor concentración de corticoides en sangre, que pueden reducir la permeabilidad intestinal de las Igs o la respuesta natural al amamantamiento (Stott et al., 1976; Shearer et al., 1992).

\section{Conclusiones}

- En las localidades de Cañete y Végueta, Lima, se presenta con mayor severidad y duración el estrés calórico en las vaquillas lecheras que en otras localidades de la zona.

- El estrés calórico afecta en menor grado a las terneras, habiendo encontrado su presencia en la localidad de Cañete.

- Los efectos adversos del estrés calórico se pueden estar presentando con moderada intensidad en las terneras y vaquillas de los establos lecheros del departamento de Lima.

\section{Agradecimiento}

Los autores manifiestan su agradecimiento al Servicio Nacional de Meteorología e Hidrografía (SENAMHI) por la información meteorológica facilitada para el desarrollo de esta investigación.

\section{Literatura Citada}

1. Amundson JL, Mader TL, Rasby RJ, Hu QS. 2006. Environmental effects on pregnancy rate in beef cattle. J Anim Sci 84: 3415-3420. doi: 10.2527/jas.2005-611
2. Aréchiga-Flores CF, Hansen PJ. 2003. Efectos climáticos adversos en la función reproductiva de los bovinos. Vet Zacatecas 2: 89-107.

3. Arias RA, Mader TL. 2010. Determination of potential risk of heat stress of cattle in four locations of central and southern Chile. Arch Med Vet 42: 33-39. doi: 10.4067/S0301732X2010000100005

4. Bolocan E. 2009. Effects of heat stress on sexual behavior in heifers. Zootehnie Biotehnologii 42: 141-148.

5. Brown-Brandl T, Chitko-McKown G, Eigenberg R, Mayer J, Welsh T, Davis J, Purswell J. 2016. Physiological responses of feedlot heifers provided access to different levels of shade. Animal. doi: 10.1017/S1751731116002664

6. Contreras MA. 2009. Efecto del estrés calórico sobre la producción en un establo lechero de la zona de Trujillo. Tesis de Ingeniero Zootecnista. Lima: Univ Nacional Agraria La Molina. 26 p.

7. Domínguez CE. 2008. Efectos del estrés calórico en vacas lecheras de Lima. Tesis de Ingeniero Zootecnista. Lima: Univ Nacional Agraria La Molina. 37 p.

8. Pereira AM, Titto EL, Infante P, Titto CG, Geraldo AM, Alves A, Leme TM, et al. 2014. Evaporative heat loss in Bos taurus: do different cattle breed scope with heat stress in the same way? J Thermal Biol 45: 87-95. doi: 10.1016/ j.jtherbio.2014.08.004

9. Healy AA, House JK, Thomson PC. 2013. Artificial insemination field data on the use of sexed and conventional semen in nulliparous Holstein heifers. J Dairy Sci 96: 1905-1914. doi: 10.3168/ jds.2012-5465

10. Hossein M, Mirzaei-Alamouti HR, Amanlou H, Mahjoubi E, Nabipour A, Aghaziarati N, Baumgard LH. 2016. Effects of heat stress on metabolism, digestibility, and rumen epithelial characteristics in growing Holstein calves. J Anim Sci 94: 77-89. doi: $10.2527 /$ jas 2015-9364 
11. [INEI] Instituto Nacional de Estadística e Informática. 2012. IV Censo Nacional Agropecuario 2012. (Internet]. Disponible en: http://desa.inei.gob.pe/ cenagro/redatam/? id=censosnacionales\#

12. Jiménez LM. 2005. Dossier: estrés por calor en vacas de leche. Revista Frisona 171 : 102-107. [Internet]. Disponible en: http://www.revistafrisona.com/Portals/0/ articulos/n171/A17105.pdf

13. Katschinski DM. 2004. On heat and cells and proteins. News Physiol Sci 19: 11-15. doi: 10.1152/nips.01403.2002

14. Mader TL, Davis MS, Brown-Brandl T. 2006. Environmental factors influencing heat stress in feedlot cattle. J Anim Sci 84: 712-719. doi: 10.2527/ 2006.843712x

15. Martínez A. 2006. Efectos climáticos sobre la producción del vacuno lechero: estrés de calor. REDVET 7(10). [Internet]. Disponible en: http:// www.veterinaria.org/revistas/redvet/ n101006/100618.pdf

16. Mellado M, Sepúlveda E, MaciasCruz U, Avendaño L, Garcia J, Veliz F, Rodríguez A. 2014. Effects of month of breeding on reproductive efficiency of Holstein cows and heifers inseminated with sex-sorted or conventional semen in a hot environment. Trop Anim Health Prod 46: 265-269. doi: 10.1007/s11250013-0470-8

17. Mohammed HO, Shearer JK, Brenneman JS. 1991. Transfer of immunoglobulins and survival of newborn calves. Cornell Vet 81: 173-182.

18. Mujibi FD, Moore SS, Nkrumah DJ, Wang Z, Basarab JA. 2010. Season of testing and its effect on feed intake and efficiency in growing beef cattle. $\mathrm{J}$ Anim Sci 88: 3789-3799. doi: 10.2527/ jas.2009-2407

19. Muroya C. 2006. Las sombras, los corrales y el estrés calórico. Lima: Gloria SA. $119 \mathrm{p}$.

20. Oikawa T. 2016. Effect of heat stress on age at first calving of Japanese Black cows in Okinawa. Anim Sci J 88: 439444. doi: 10.1111/asj. 12669
21. Putney DJ, Malayer JR, Gross TS, Thatcher WW, Hansen PJ, Drost M. 1988. Heat stress-induced alterations in the synthesis and secretion of proteins and prostaglandins by cultured bovine conceptus and uterine endometrium. Biol Reprod 39: 717-728.

22. Ravagnolo O, Misztal I, Hoogenboom G. 2000. Genetic component of heat stress in dairy cattle, development of heat index function. J Dairy Sci 83: $2120-$ 2125. doi: 10.3168/jds.S00220302(00)75094-6

23. Rodríguez L, Ara M, Huamán H, Echevarría L. 2005. Modelos de ajuste para curvas de lactación de vacas en crianza intensiva en la cuenca de Lima. Rev Inv Vet Perú 16: 1-12. doi: 10.15381/ rivep.v16il.1517

24. Roland L, Drillich M, Klein-Jöbstl D, Iwersen M. 2016. Invited review: influence of climatic conditions on the development, performance, and health of calves. J Dairy Sci 99: 2438-2452. doi: 10.3168/jds.2015-9901

25. Sandoval R, Ruiz L, Carcelén F. 2017. Determinación de la tasa de servicio y de los factores que la afectan en los establos lecheros intensivos de Lima, Perú. Rev Inv Vet Perú 28: 314-326. doi: 10.15381/rivep.v28i2.13081

26. Shearer J, Mohammed HO, Brenneman JS, Tran TQ._1992. Factors associated with concentrations of immunoglobulins in calostrum at the first milking post-calving. Prev Vet Med 14: 143-154. doi: 10.1016/01675877(92)90091-S

27. Stott GH, Wiersma F, Menefee BE, Radwanski R. 1976. Influence of environmental of passive immunity in calves. J Dairy Sci 59: 1306-1311. doi: 10.3168/jds.S0022-0302(76)84360-3

28. St-Pierre NR, Cobanov B, Schnitkey G 2003. Economic losses from heat stress by US livestock industries. J Dairy Sci 86(Suppl): E52-E77. doi: 10.3168/ jds.S0022-0302(03)74040-5 
29. Thatcher $W W$. 1974. Effects of season, climate, and temperature on reproduction and lactation. J Dairy Sci 57: 360-368. doi: 10.3168/jds.S0022-0302(74)84894-0

30. Webster AJF. 1983. Environmental stress and the physiology, performance and health of ruminants. J Anim Sci 57: 1584-1593. doi: $10.2527 / \mathrm{j}$ as 1983 . $5761584 \mathrm{x}$

31. West JW. 2003. Effects of heat-stress on production in dairy cattle. J Dairy Sci 86: 2131-2144. doi: 10.3168/jds.S00220302(03)73803-X

32. West JW, Mullinix BG, Bernard JK. 2003. Effects of hot, humid weather on milk temperature, dry matter intake, and milk yield of lactating dairy cows. J Dairy Sci 86: 232-242. doi: 10.3168/jds.S00220302(03)73602-9
33. Wheelock JB, Rhoads RP, Vanbaale MJ, Sanders SR, Baumgard LH. 2010. Effects of heat stress on energetic metabolism in lactating Holstein cows. J Dairy Sci 93: 644-655. doi: 10.3168/ jds.2009-2295

34. Wilson SJ, Kirby CJ, Koenigsfeld AT, Keisler DH, Lucy MC. 1998. Effects of controlled heat stress on ovarian function of dairy cattle. 2. Heifers. J Dairy Sci 81: 2132-2138. doi: 10.3168/ jds.S0022-0302(98)75789-3

35. Wise ME, Armstrong DV, Huber JT, Hunter R, Wiersma F. 1988. Hormonal alterations in the lactating dairy cow in response to thermal stress. J Dairy Sci 71: 2480-2485. doi: 10.3168/jds.S00220302(88)79834-3 